\title{
Cardiac cephalalgia: one case with cortical hypoperfusion in headaches and literature review
}

\author{
Miao Wang ${ }^{1 \dagger}$, Lu Wang $^{2 \dagger}$, Changfu Liu ${ }^{3}$, Xiangbing Bian ${ }^{4}$, Zhao Dong ${ }^{5^{*}}$ and Shengyuan $\mathrm{Yu}^{5}$
}

\begin{abstract}
Background: Cardiac cephalalgia (CC) is a rare disease occurring during an episode of myocardial ischemia and relieved by nitroglycerine. Though more than 30 cases of CC have been reported since 1997, the mechanism is yet obscure. Herein, a case of CC is presented and discussed in relevance with previous literature to propose a novel hypothesis about the mechanism of CC.

Method: A CC patient with cortical hypoperfusion during headache attacks was presented, which has never been reported. All published cases of CC via PubMed (http://www.ncbi.nlm.nih.gov/pubmed) in English literature, between 1997 and 2016, were reviewed.

Results: A patient suffering from CC presented a cerebral hypoperfusion during a headache attack. This phenomenon had not been observed since CC was introduced in 1997. The literature review summarized the clinical presentations, neuroimaging features, ECG, and coronary angiography features of 35 CC patients.

Conclusion: Based on the phenomenon of hypoperfusion in the event of a headache, the vessel constriction hypothesis was proposed including two potential physiological mechanisms underlying the pathophysiology of CC.
\end{abstract}

Keywords: Cardiac cephalalgia, Clinical features, Neuroimages, Pathophysiology

\section{Introduction}

Headaches associated with exertion or sexual activities have been regarded as benign if structural lesions can be excluded. In 1997, Lipton et al. summarized two current and five previous cases of an exertional headache complicated with acute coronary syndrome and discovered that the headache was relieved by treatments for acute coronary syndrome, such as the administration of nitroglycerine and/or surgical interventions including coronary artery bypass grafting or percutaneous angioplasty [1]. Thus, they deemed it a rare type of an exertional headache and suggested the term "cardiac cephalalgia" (CC) describing the type of headache, which may have life-threatening implications if misdiagnosed. Since 1997, more than 20 reports of $\mathrm{CC}$ have been reported; however, the

\footnotetext{
*Correspondence: dong_zhaozhao@126.com

${ }^{\dagger}$ Equal contributors

${ }^{5}$ Department of Neurology, Chinese PLA General Hospital, Fuxing Road 28,

Haidian District, Beijing 100853, China

Full list of author information is available at the end of the article
}

pathogenesis remains unclear. Hitherto, three hypotheses were proposed to illustrate the mechanism of CC: convergence of nerve fibers within the spinal cord, increased intracranial pressure secondary to decreased venous return from the brain, and increased inflammatory mediators causing vasodilation. The present paper aims to delineate the clinical features of $\mathrm{CC}$ and put forth a prospective mechanism.

\section{Materials and methods Case and literature review We described the clinical features as well as the neuro- imaging data of CC patients, and searched PubMed database using the terms "cardiac cephalalgia", "cardiac cephalgia", "headache and angina", "headache and acute coronary syndrome", and "headache and myocardial in- farction". The following limitations were exercised: full text, English language only, and published after 1997.}




\section{Results}

\section{Case presentation}

A 40-year-old male presented a 4-year history of episodic bitemporal headaches before he was seen for neurological consultation in outpatient. The headaches were rated as 7-10 in severity on the visual analog scale, pulsatile, tight in quality, and occasionally radiating to upper limbs. The headaches were sometimes also associated with chest discomfort, palpitations, cold sweating, and facial pallor. However, the patient denied nausea, vomiting, photophobia, and phonophobia.

The symptoms attack occurred 2-3 times per month, elicited by exertion, cold stimuli, and sexual activities, lasting 5-10 $\mathrm{min}$, and relieved after treatment with nitrates. Coughing, sneezing, or having a bowel movement did not trigger the pain.

The patient self-administered aspirins and statins postdiagnosis of acute non-ST-elevation myocardial infarction (NSTEMI) in 2009 and nifedipine to control hypertension since 2001. Additionally, he presented 20 years of smoking history with 30 cigarettes/day but has ceased smoking for 5 years before the start of headache.

Physical examination revealed normal blood pressure, heart rate and rhythm, systemic and neurological examination results. The cardiac enzymes were in normal range at the time of headache attack. The estimation of catecholamines and their metabolites were normal. The ECG showed inverted T wave (Fig. 1).

The patient underwent brain MR examinations with routine clinical sequences including axial T1W, T2 FLAIR, diffusion-weighted imaging (DWI), and MRA
(Magnetic Resonance Angiography) on a 3.0 T MR system (Discovery MR750, GE Healthcare, Milwaukee, WI, USA) equipped with an 8-channel head coil to receive signals. Perfusion-weighted images (PWI) were obtained using a 3D pCASL technique.

T1, T2, FLAIR and DWI weighted images of brain MRI were negative (Fig. 2a). However, the PWI revealed cerebral hypoperfusion during the headache attacks (Fig. 2b, c).

Owing to the headaches provoked by exertion, cold stimuli, and sexual activities and relieved after administration of nitrates, CC diagnosis should be suspected according to the international classification of headache disorder (ICHD-3ß) in 2013 [2]. Thus, a coronary angiography was performed, which demonstrated complete occlusion at the middle segment of left anterior descending (LAD) and proximal right coronary arteries (RCA), $80 \%$ stenosis at the middle segment of left circumflex (LCX), and $80 \%$ stenosis at the bifurcation of the first diagonal (Fig. 3). Percutaneous transluminal coronary angiography (PTCA), stenting of LAD, and bifurcation of the first diagonal and the RCA were carried out successfully. Six months following the operation, the patient did not report any recurrence of a headache.

\section{Literature review}

The demographic data and the clinical manifestations of the 35 cases were indicated in Tables 1 and 2. A male predominance was observed (male:female ratio 1.5:1). The mean age was 62-years-old.

The clinical manifestations of the headache were displayed in Table 1. In more than half of the patients, the

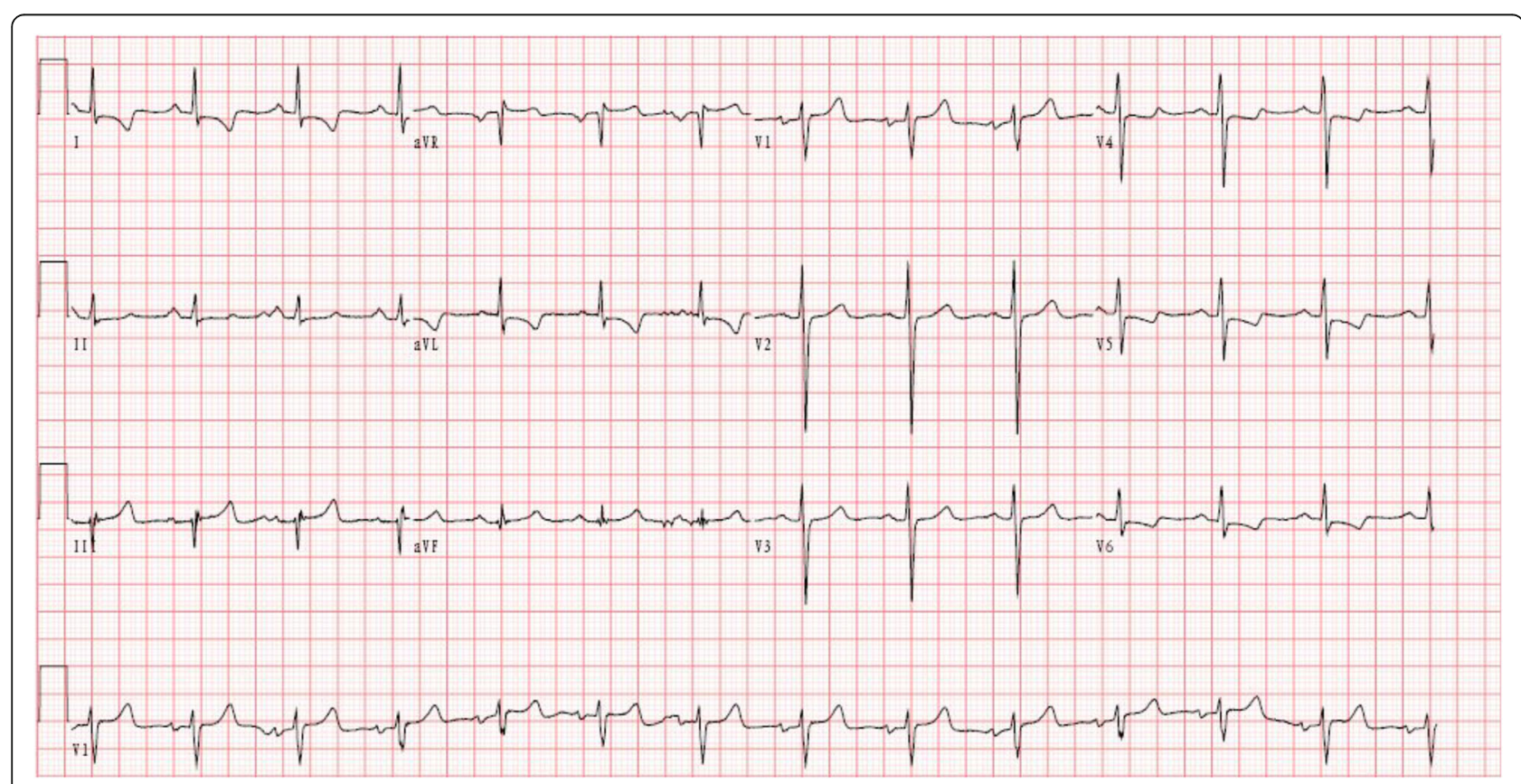

Fig. 1 The ECG showed inverted T wave 


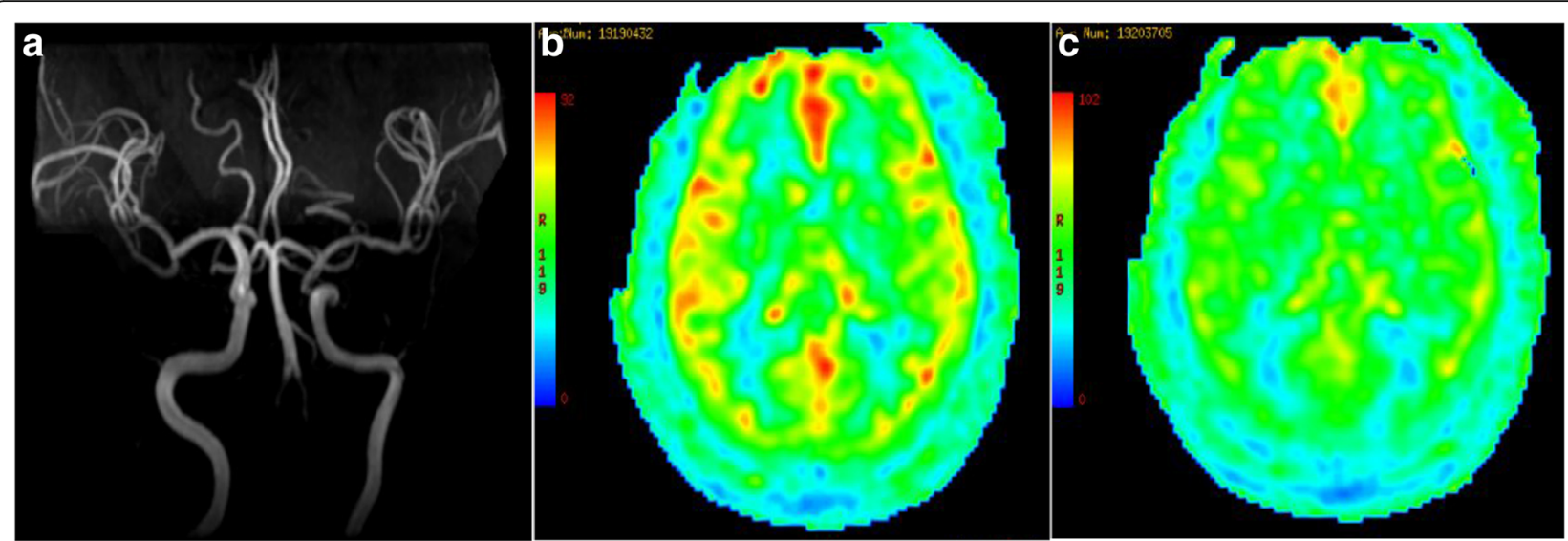

Fig. 2 MRA (a) was negative when headache attack. PWI obtained during the headache not attack (b). PWI obtained during headache attack (c)

headache could be trigged in conditions with high myocardial oxygen consumption, such as exertion, sexual activity, and emotional fluctuation. However, 6 cases have been reported; wherein the headache appeared during rest. The symptom was not localized in a specific area but involved frontal, temporal, parietal, and occipital regions. Moreover, the headache may be unilateral or bilateral. The quality of a headache was varied including shooting, bursting, dull, and squeezing. A maximum number of patients had a severe intensity of headache, and only two patients presented mild or moderate. In some cases, the headache had no accompanying symptoms while in others, it was accompanied by photophobia, phonophobia, osmophobia, and nausea or vomiting. Notably, all the reviewed cases of CC described the resolution of a headache after reinstating the flow in cardiac vessels by medical or surgical interventions.

The clinical manifestations of cardiac ischemia were illustrated in Table 2. About $1 / 2$ of the CC patients (18 cases) were without typical angina symptoms, which included chest pain or tightness, palpitations, and dyspnea. Most of the patients showed pathological alterations of the baseline ECG trace, such as ST-segment elevations or depressions and T-wave inversions, as well as, elevated cardiac enzymes.
However, three separate cases presented normal ECG at rest $[3,4]$, and one case presented a negative ECG even under stress [5]. Thirty-one out of 35 cases underwent coronary artery stenosis or occlusion. However, normal coronary angiography results could not exclude the diagnosis of $\mathrm{CC}$ as two cases continued to present normal coronary angiography $[4,6]$. After intra-arterial injection of acetylcholine in 1 of the cases, coronary artery's contraction was observed. These two cases were suggested as potential variants of angina.

\section{Discussion}

\section{Clinical features}

In "typical" cases that were triggered by exertion or sexual activity or emotion fluctuation and accompanied by the symptoms of angina, a diagnosis of CC could be established according to the medical history demonstrating the exact onset of headache concurrently with acute myocardial ischemia, abnormality of ECGs performed at rest or under stress, elevating cardiac markers (CPK-MB, myoglobin, and troponin) and coronary angiography illustrating coronary arteries occlusion or stenosis. However, the majority of cases were "atypical". Headaches may occur as the sole symptom without the symptoms of angina,

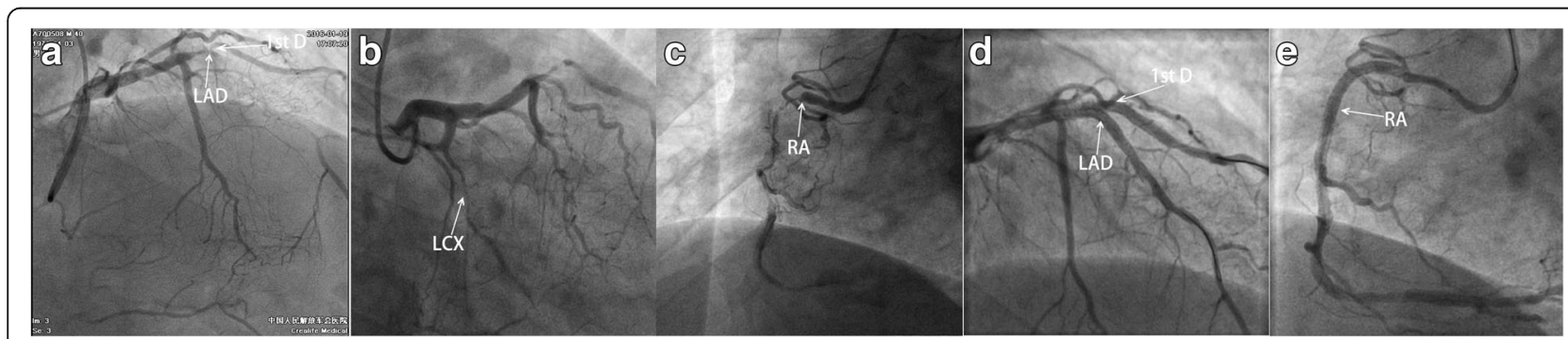

Fig. 3 Coronary angiography demonstrated complete occlusion at the middle segment of left anterior descending (LAD) and proximal right coronary arteries (RCA), 80\% stenosis at the middle segment of left circumflex (LCX), and 80\% stenosis at the bifurcation of the first diagonal (a, b, c). Percutaneous transluminal angiography (PTCA), stenting of LAD, and bifurcation of the first diagonal and the RCA were carried out successfully (d, e) 
Table 1 Clinical manifestations of cardiac cephalalgia headache

\begin{tabular}{|c|c|}
\hline \multicolumn{2}{|l|}{ A. Sexual characteristic } \\
\hline$M / F$ & $21 / 14$ \\
\hline \multicolumn{2}{|l|}{ B. Triggers } \\
\hline Expertise/Sexual/Emotion & 18 \\
\hline None & 6 \\
\hline Not mentioned & 11 \\
\hline \multicolumn{2}{|l|}{ C. Side of headache } \\
\hline Right & 3 \\
\hline Left & 2 \\
\hline Bilateral & 16 \\
\hline Not mentioned & 14 \\
\hline \multicolumn{2}{|l|}{ D. Regions of headache } \\
\hline Occipital & 16 \\
\hline Vertex & 6 \\
\hline Frontal & 7 \\
\hline Temporal & 5 \\
\hline Parietal & 3 \\
\hline \multicolumn{2}{|l|}{ Other Regions ${ }^{\mathrm{a}}$} \\
\hline \multicolumn{2}{|l|}{ E. Quality of headache } \\
\hline Shooting & 4 \\
\hline Bursting & 4 \\
\hline Sharp & 5 \\
\hline Dull & 6 \\
\hline Squeezing & 2 \\
\hline Pulsating & 2 \\
\hline Not mentioned & 16 \\
\hline \multicolumn{2}{|l|}{ F. Intensity of headache } \\
\hline \multicolumn{2}{|c|}{$\begin{array}{l}33 \text { cases presented severe intensity. } 2 \text { cases presented mild or } \\
\text { moderate }\end{array}$} \\
\hline \multicolumn{2}{|l|}{ G. Associations with headache } \\
\hline Nausea or Vomiting & 10 \\
\hline Sonophobia & 1 \\
\hline Photophobia & 1 \\
\hline Sweating & 5 \\
\hline Dizziness & 2 \\
\hline \multicolumn{2}{|l|}{ Other Associations ${ }^{b}$} \\
\hline Without & 21 \\
\hline
\end{tabular}

Other Regions $\mathrm{s}^{\mathrm{a}}: 2$ cases radiate to shoulders. 2 case presented full head pain. 1 case presented right eyeball pain. 1 case had not mentioned Other Associations ${ }^{\mathrm{b}}: 1$ case associated with Confusion, agitation

absence of triggers, absence of ECG abnormalities (Table 2) when acute myocardial ischemia onset. In "atypical" cases, the doctors might be prone to omit the cardiac examinations (cardiac marker and coronary angiography) and make an incorrect diagnosis of benign headaches related to exertion, cough, migraine, and even orgasm. Especially, when some cases resembled migraine without aura, we should be able to make a diagnosis and prescribe triptans, which are vasoconstrictors aggravating the cardiac ischemic. However, there were more clinical clues implying the diagnosis of $\mathrm{CC}$. We observed that most of the patients were above 50 years of age, and the majority of them presented cardiac risk factors such as hypertension, hyperlipidemia, diabetes, and smoking. Therefore, it is suggested that the patients suffering a new headache, who are over 50-years-old or display cardiac risk factors, should be suspected of CC. We also found that the headaches of all the patients reacted to vasodilators. Also, we demonstrated that the experimental treatment by vasodilators might be an efficient method in the event of difficulty in differentiating $\mathrm{CC}$ from other headaches. Moreover, even the patient showed a normal ECG; thus, the diagnosis of $\mathrm{CC}$ should not be excluded, because CC patients could have a normal ECG even under the stress test $[3,4]$. Therefore, we suggested that coronary angiography should be recommended to all patients with suspected $\mathrm{CC}$ for accurate diagnosis.

\section{Pathophysiology}

Since the diagnosis had been introduced in 1997, several theories have been proposed about the pathogenesis of CC. Based on the current case, we proposed a new hypothesis about the mechanisms of CC vessel constriction hypothesis.

In the current case, the PWI of brain MRI confirmed the cerebral hypoperfusion; however, MRA was normal during the headache attack, which had not been reported. Thus, this phenomenon might lead to headache by two possible physiological mechanisms. Firstly, the hypoperfusion may suggest reversible microvessels constriction during headache. The reversible cerebral vasoconstriction syndrome (RCVS) has been classified with moderate to a severe headache, which was also accompanied by reversible vasoconstriction of the cerebral vasculature [2]. Since the reversible vessels constriction could be restored both in CC and RCVS, we speculated that they might share some common mechanisms. Several previous studies revealed that vasoconstriction of RCVS was associated with sympathetic over-activity based on clinical observations or hypotheses including central vascular tone changes, aberrant sympathetic response, pheochromocytoma, and autonomic dysreflexia [7-10]. The mechanism of CC might also be linked to sympathetic hyperfunction due to the following reason: when myocardial ischemia occurred, the cardiac sympathetic afferents nerve could be stimulated. Hence, the activation of sympathetic afferents nerve could increase the sympathetic outflow through cardiac sympathetic nerve reflexes, which has been confirmed by several physiological tests [11-13]. 


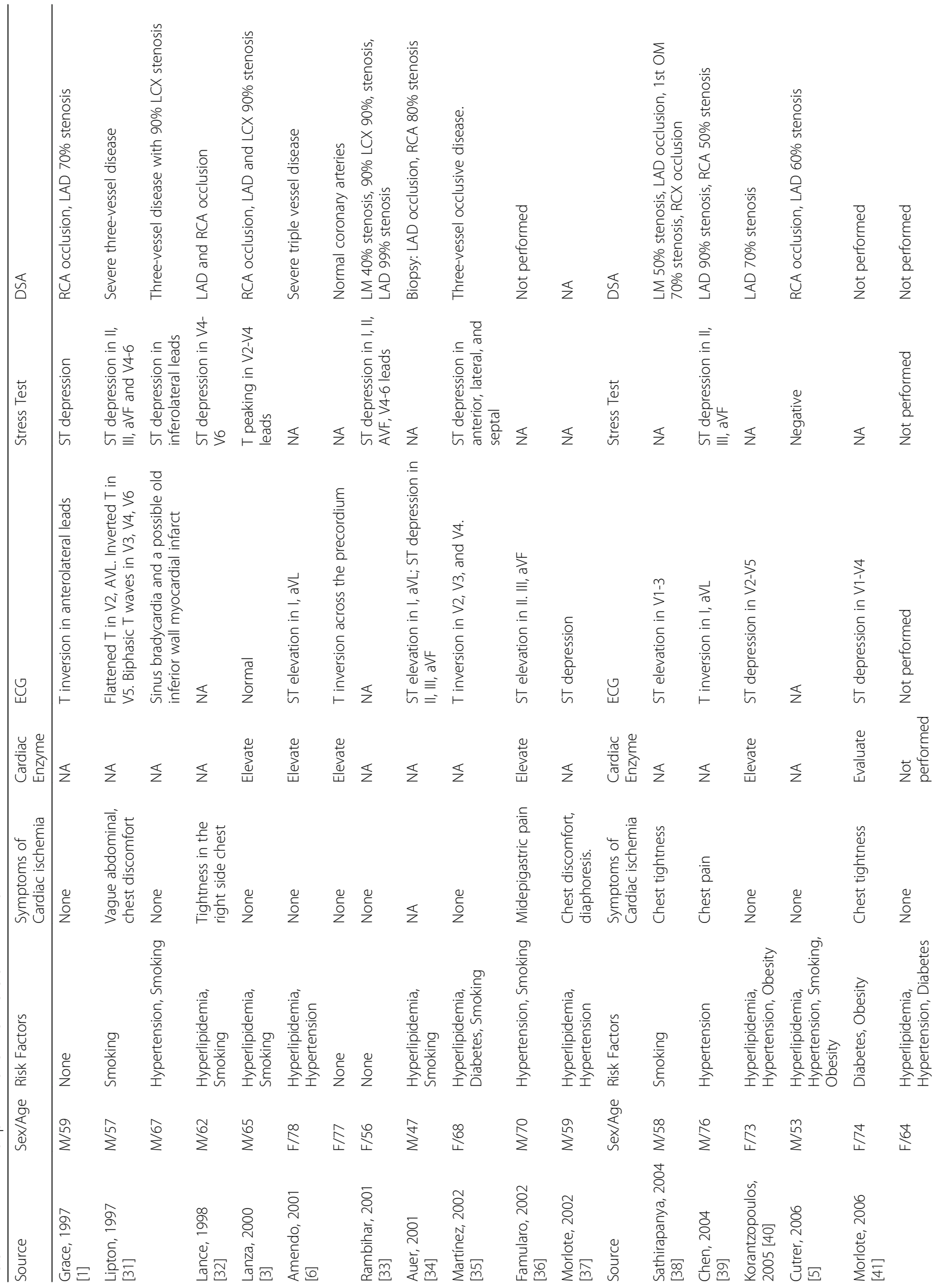




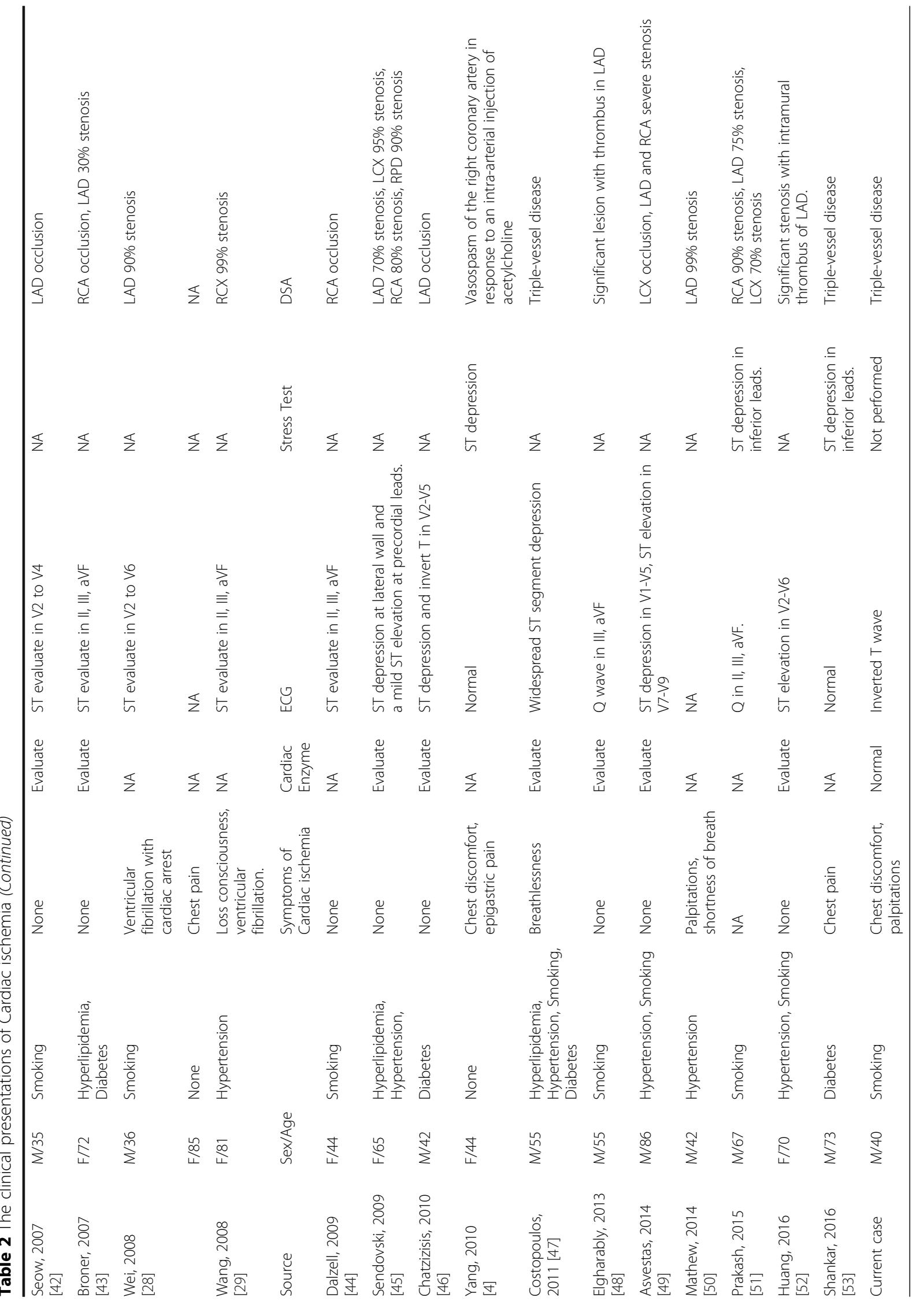


On the other hand, sympathetic hyperfunction and parasympathetic hypofunction were found in migraine patients $[14,15]$. And the autonomic nervous system imbalance might be derived from the abnormal functional connections between the hypothalamus and other brain structures involved in autonomic function including the brain stem [16]. Thus, we speculated that the sympathetic hyperfunction in CC patients might also be associated with abnormal hypothalamic functional connectivity. Although the distinguishing characteristics of RCVS were attributed to constriction of medium- and large-sized arteries, the extent of headache and vasoconstriction in RCVS was asynchronous [17-19]. This implicated that the headache might not be derived from medium- or large-sized arteries constriction. Ducros et al. demonstrated that headache could onset 1 week before vasoconstriction of large- and medium-sized arteries appeared, and in the same period the complications of cortical subarachnoid hemorrhage (cSAH), intracerebral hemorrhage (ICH), and reversible posterior leukoencephalopathy syndrome (RPLS) were observed. The ischemic events, including TIAs and cerebral infarction, often occurred after approximately 7 days. Based on the temporal pattern, their study illustrated that the underlying disturbance in the control of cerebral arterial tone first involved small distal arteries responsible for hemorrhages and RPLS and then progressed towards medium- and large-sized arteries responsible for ischemic events. Furthermore, the study also speculated that the headache of RCVS might primarily be due to the involvement of small distal arteries, with sudden changes in caliber (constriction or dilatation) that could stimulate perivascular pain-sensitive fibers $[18,20]$. Thus, we inferred that myocardial ischemia might activate the sympathetic system, causing small intracranial arteries constriction and leading to a headache attack. Secondly, the cortical hypoperfusion might induce the occurrence of cortical spreading depression (CSD) in primary headaches, especially in a migraine [21]. Several experiments showed that CSD could contribute to the headache via activating meningeal nociceptors $[22,23]$ and activating or disinhibiting the second-order neurons in the trigeminocervical complex (TCC) [22, 24, 25]. Hence, according to the phenomenon of cerebral hypoperfusion, we proposed another possible mechanism: intracranial arteries constriction derived from myocardial ischemia lead to a CSD, which in turn caused a headache.

The field also proposed several other hypotheses to illustrate the mechanisms of $\mathrm{CC}$. The first hypothesis was the convergence projection mechanism based on the fact that visceral afferent nerves from the heart and somatic afferent nerves from the head converged in the same spinal segments ( $\mathrm{C} 1$ and $\mathrm{C} 2$ segments) belonging to TCC [26, 27]; when cardiac ischemic occurred, TCC was activated, leading to headache, similar to the reference pain of angina.
The second hypothesis is hyper intracranial pressure mechanism: the sudden reduction of cardiac output associated with cardiac ischemia increased pressure in the left ventricle and in the right atrium, which might cause a decrease in venous return from the brain and subsequently an elevation of intracranial pressure, causing the headache $[1,4,28,29]$. The third hypothesis is neurochemical mediator mechanism: during cardiac ischemia, several chemical mediators such as bradykinin, histamine, and substance $\mathrm{P}$ are released into the blood. These vasodilators could give rise to headache by dilation of the cerebrovasculature $[1,30]$. However, these hypotheses were contrary to the fact that nitrates, also vasodilators, could relieve CC. Moreover, the PWI in the current case suggested microvessels constriction rather than vessel dilation during headache, which was also converse to this hypothesis.

\section{Conclusions}

We presented a CC patient with cortical hypoperfusion during headache attack, which had never been reported. Based on the phenomenon, we proposed vessel constriction hypothesis including two possible physiological mechanisms to explicate the pathophysiology of CC. Firstly, when myocardial ischemia occurred, the sympathetic system was activated causing small intracranial arteries constriction and leading to a headache, similar to the mechanisms of RCVS. Secondly, hypoperfusion might induce CSD that might contribute to the headache confirmed by several experiments.

\section{Abbreviations \\ CC: Cardiac cephalalgia; CSAH: cortical subarachnoid hemorrhage: CSD: Cortical spreading depression; DWI: Diffusion-weighted imaging; ECG: Electrocardiogram; ICH: Intracerebral hemorrhage; LAD: Left anterior descending; LCX: Left circumflex; MRA: Magnetic resonance angiography; NSTEMI: Non-ST-elevation myocardial infarction; PTCA: Percutaneous transluminal coronary angiography; PWI: Perfusion-weighted images; RCA: Right coronary arteries; RCVS: Reversible cerebral vasoconstriction syndrome; RPLS: Reversible posterior leukoencephalopathy syndrome; TCC: Trigeminocervical complex}

\section{Funding}

This work was supported by the National Scientific Research Fund of China (grant number 81471146); the National Scientific Research Fund of China (grant number 81471147); the Capital Development Scientific Research (grant number 2014-4-5013).

\section{Authors' contributions \\ MW and LW performed literature review, extracted the patient journal, and drafted the manuscript. CL contributed in coronary angiography. XB contributed in Neuroimages. ZD and SY contributed in revising the manuscript. All authors read and approved the final manuscript.}

\section{Competing interests}

The authors declare that they have no competing interests.

Consent for publication

Written informed consent was obtained from the patient for the publication of this report and any accompanying images. 


\section{Author details}

The Department of Geriatric Neurology, Chinese PLA General Hospital, Beijing, China. ${ }^{2}$ The Outpatient Department of Fuxing Road No. 7, the First Affiliated Hospital of PLA General Hospital, Beijing, China. ${ }^{3}$ The Department of of Cardiology, Chinese PLA General Hospital, Beijing, China. ${ }^{4}$ The Department of of Radiology, Chinese PLA General Hospital, Beijing, China. 5Department of Neurology, Chinese PLA General Hospital, Fuxing Road 28, Haidian District, Beijing 100853, China.

\section{Received: 15 December 2016 Accepted: 7 February 2017}

\section{Published online: 20 February 2017}

\section{References}

1. Grace A, Horgan J, Breathnach K et al (1997) Anginal headache and its basis. Cephalalgia 17(3):195-196

2. Headache Classification Committee of the International Head-ache Society (IHS) (2013) The international classification of headache disorders, 3rd edition (beta version). Cephalalgia 33:629-808

3. Lanza GA, Sciahbasi A, Sestito A, Maseri A (2000) Angina pectoris: a headache. Lancet 356:998

4. Yang $Y$, Jeong D, Jin DG et al (2010) A case of cardiac cephalalgia showing reversible coronary vasospasm on coronary angiogram. J Clin Neurol 6(2): 99-101

5. Cutrer FM, Huerter K (2006) Exertional headache and coronary ischemia despite normal electrocardiographic stress testing. Headache 46(1):165-167

6. Amendo MT, Brown BA, Kossow LB et al (2001) Headache as the sole presentation of acute myocardial infarction in two elderly patients. Am J Geriatr Cardiol 10(2):100-101

7. Chen SP, Fuh JL, Wang SJ (2011) Reversible cerebral vasoconstriction syndrome: current and future perspectives. Expert Rev Neurother 11:1265-1276

8. Dodick DW (2002) Thunderclap headache. J Neurol Neurosurg Psychiatry 72:6-11

9. Gerretsen P, Kern RZ (2009) Reversible cerebral vasoconstriction syndrome: a hunderclap headache-associated condition. Curr Neurol Neurosci Rep 9: $108-114$

10. Edvardsson B, Persson S (2010) Reversible cerebral vasoconstriction syndrome associated with autonomic dysreflexia. J Headache Pain 11:277-280

11. Minisi AJ, Thames MD (1993) Distribution of left ventricular sympathetic afferents demonstrated by reflex responses to transmural myocardial ischemia and to intracoronary and epicardial bradykinin. Circulation 87:240-246

12. Webb SW, Adgey AA, Pantridge JF (1972) Autonomic disturbances at onset of acute myocardial infarction. Br Med J Clin Res 3:89-92

13. Randall WC, Hasson DM, Brady JV (1978) Acute cardiovascular consequences of anterior descending coronary artery occlusion in unanesthetized monkey. Proc Soc Exp Biol Med 58:135-140

14. Appel S, Kurityzi A, Zahavi I, Zigelman M (1992) Evidence for instability of the autonomic nervous system in patients with migraine. Headache 32:10-17

15. Schecter A, Stewart WF, Silberstein SD et al (2002) Migraine and autonomic nervous system function: a population based case control study. Neurology 58:422-427

16. Moulton EA, Becerra L, Johnson A et al (2014) Altered hypothalamic functional connectivity with autonomic circuits and the locus coeruleus in migraine. PLoS One 9, e95508

17. Chen SP, Fuh JL, Chang FC, Lirng JF, Shia BC, Wang SJ (2008) Transcranial color doppler study for reversible cerebral vasoconstriction syndromes. Ann Neurol 63(6):751-757

18. Ducros A, Fiedler U, Porcher R, Boukobza M, Stapf C, Bousser MG (2010) Hemorrhagic manifestations of reversible cerebral vasoconstriction syndrome: frequency, features, and risk factors. Stroke 41(11):2505-2511

19. Chen SP, Fuh JL, Wang SJ et al (2010) Magnetic resonance angiography in reversible cerebral vasoconstriction syndromes. Ann Neurol 67(5):648-656

20. Ducros A, Boukobza M, Porcher R, Sarov M, Valade D, Bousser MG (2007) The clinical and radiological spectrum of reversible cerebral vasoconstriction syndrome. A prospective series of 67 patients. Brain 130:3091-3101

21. Dalkara T, Nozari A, Moskowitz MA (2010) Migraine aura pathophysiology: the role of blood vessels and microembolisation. Lancet Neurol 9:309-317

22. Moskowitz MA, Nozaki K, Kraig RP (1993) Neocortical spreading depression provokes the expression of c-fos protein-like immunoreactivity within trigeminal nucleus caudalis via trigeminovascular mechanisms. J Neurosci 13:1167-1177

23. Zhang X, Levy D, Noseda R, Kainz V, Jakubowski M, Burstein R (2010) Activation of meningeal nociceptors by cortical spreading depression: implications for migraine with aura. J Neurosci 30:8807-8814
24. Zhang X, Levy D, Kainz V, Noseda R, Jakubowski M, Burstein R (2011) Activation of central trigeminovascular neurons by cortical spreading depression. Ann Neurol 69:855-865

25. Borysovych BV, Bogdanova OV, Lombard A (2015) Cortical spreading depression decreases Fos expression in rat periaqueductal gray matter Neurosci Lett 12(585):138-143

26. Foreman RD, Garrett KM, Blair RW (2015) Mechanisms of cardiac pain. Compr Physiol 5(2):929-960

27. Akerman S, Romero-Reyes M (2013) Insights into the pharmacological targeting of the trigeminocervical complex in the context of treatments of migraine. Expert Rev Neurother 13(9):1041-1059

28. Wei JH, Wang HF (2008) Cardiac cephalalgia: case reports and review. Cephalalgia 28(8):892-896

29. Wang WW, Lin CS (2008) Headache angina. Am J Emerg Med 26(3):387

30. Kuo DC, Oravitz JJ, DeGroat WC (1984) Tracing of afferent and efferent pathways in the left inferior cardiac nerve of the cat using retrograde and transganglionic transport of horseradish peroxidase. Brain Res 321:111-118

31. Lipton RB, Lowenkopf T, Bajwa ZH et al (1997) Cardiac cephalgia: a treatable form of exertional headache. Neurology 49(3):813-816

32. Lance JW, Lambros J (1998) Unilateral exertional headache as a symptom of cardiac ischemia. Headache 38(4):315-316

33. Rambihar VS (2001) Headache angina. Lancet 357(9249):72

34. Auer J, Berent R, Lassnig E, Eber B (2001) Headache as a manifestation of fatal myocardial infarction. Neurol Sci 22:395-397

35. Martínez HR, Rangel-Guerra RA, Cantú-Martínez L et al (2002) Cardiac headache: hemicranial cephalalgia as the sole manifestation of coronary ischemia. Headache 42(10):1029-1032

36. Famularo G, Polchi S, Tarroni P (2002) Headache as a presenting symptom of acute myocardial infarction. Headache 42(10):1025-1028

37. Gutiérrez-Morlote J, Pascual J (2002) Cardiac cephalgia is not necessarily an exertional headache: case report. Cephalalgia 22(9):765-766

38. Sathirapanya P (2004) Anginal cephalgia: a serious form of exertional headache. Cephalalgia 24(3):231-234

39. Chen SP, Fuh JL, Yu WC et al (2004) Cardiac cephalalgia. Case report and review of the literature with new ICHD-II criteria revisited. Eur Neurol 51(4): 221-226

40. Korantzopoulos P, Karanikis P, Pappa E et al (2005) Acute non-ST-elevation myocardial infarction presented as occipital headache with impaired level of consciousness-a case report. Angiology 56(5):627-630

41. Gutierrez MJ, Fernandez JM, Timiraos JJ et al (2005) Cardiac cephalgia: an under diagnosed condition? Rev Esp Cardiol 58:1476-1478

42. Seow VK, Chong CF, Wang TL et al (2007) Severe explosive headache: a sole presentation of acute myocardial infarction in a young man. Am J Emerg Med 25(2):250-251

43. Broner S, Lay C, Newman L et al (2007) Thunderclap headache as the presenting symptom of myocardial infarction. Headache 47(5):724-725

44. Dalzell JR, Jackson CE, Robertson KE et al (2009) A case of the heart ruling the head: acute myocardial infarction presenting with thunderclap headache. Resuscitation 80(5):608-609

45. Sendovski U, Rabkin Y, Goldshlak L et al (2009) Should acute myocardial infarction be considered in the differential diagnosis of headache? Eur J Emerg Med 16(1):1-3

46. Chatzizisis YS, Saravakos P, Boufidou A et al (2010) Acute myocardial infarction manifested with headache. Open Cardiovasc Med J 4:148-150

47. Costopoulos C (2011) Acute coronary syndromes can be a headache. Emerg Med J 28(1):71-73

48. Elgharably Y, Iliescu C, Sdringola S et al (2013) Headache: a symptom of acute myocardial infarction. Eur J Cardiovasc Med 11:170-174

49. Asvestas D, Vlachos K, Salachas A et al (2014) Headache: an unusual presentation of acute myocardial infraction. World J Cardiol 6(6):514-516

50. Mathew PG, Boes CJ, Garza I (2015) A tale of Two systems: cardiac cephalalgia vs migrainous thoracalgia. Headache 55(2):310-312

51. Prakash S, Panchani N, Rathore C et al (2016) Cardiac cephalalgia: first case from India. Ann Indian Acad Neurol 19(2):252-254

52. Huang CC, Liao PC (2016) Heart attack causes headache - cardiac cephalalgia. Acta Cardiol Sin 32(2):239-242

53. Shankar A, Allan CL, Smyth D et al (2016) Cardiac cephalgia: a diagnostic headache. Intern Med J 46:1219-1221 